PHYSICAL REVIEW D, VOLUME 63, 029902(E)

\title{
Erratum: Third post-Newtonian higher order ADM Hamilton dynamics \\ for two-body point-mass systems \\ [Phys. Rev. D 57, 7274 (1998)]
}

\section{Piotr Jaranowski and Gerhard Schäfer}

(Published 29 December 2000)

DOI: 10.1103/PhysRevD.63.029902

In Eq. (51), $\phi_{(3)}$ should read $\phi_{(2)}$.

In Eq. (68), the last two terms proportional to $1 / r$ must contain $(\mathbf{p} \cdot \mathbf{v})$ instead of $(\mathbf{q} \cdot \mathbf{v})$. The corrected version of these terms reads

$$
-4(\mathbf{n} \cdot \mathbf{p})^{2}(\mathbf{p} \cdot \mathbf{v})^{2}-4 \mathbf{p}^{2}(\mathbf{p} \cdot \mathbf{v})^{2}
$$

In Eq. (80), $1 /(16 \pi)^{2}$ should be replaced by $1 /(16 \pi)^{4}$. 